\title{
Multi-Temporal Aspects on Contextual Variability Modeling
}

\author{
Jorge Luis Victória Barbosa \\ Programa de Pós-Graduação em Computação Aplicada (PPGCA) \\ Universidade do Vale do Rio dos Sinos (UNISINOS) - São Leopoldo - RS - Brasil \\ jbarbosa@unisinos.br
}

\begin{abstract}
Time is one of the most relevant aspects when we model contextual variability. The temporal perspective guides the modeling of context-aware systems. Despite natural and consensual perception of time, the integrated modeling of its dimensions for developing context-aware software is a recent theme of study. The Past is stored in Contexts Histories, the Present is modeled through Profile Management and the Future is anticipated using Context Prediction. This article discusses the modeling of these three dimensions in the context-aware systems, indicates challenges to each dimension and proposes a system architecture to manage the contextual variability on multi-temporal systems. I believe that this text can be a seminal article to stimulate and guide future researches on temporal aspects of computational environments.
\end{abstract}

\section{Introduction}

Approximately 25 years ago, Weiser [1991] introduced the concept of Ubiquitous Computing. Ten years after, Satyanarayanan [2001] reinforced the concept through a more concrete perspective entitled Pervasive Computing. Also, in 2001, Dey, Abowd and Salber [2001] proposed their classical definition of Context. Currently, contextaware systems have been used in a diverse range of knowledge areas, such as, health [Vianna et al. 2014] [Vianna et al. 2019], well-being [Vianna and Barbosa 2017], competence management [Rosa et al. 2015], learning [Wagner et al. 2014] [Larentis et al. 2019], commerce [Barbosa et al. 2016], accessibility [Barbosa et al. 2018] and agriculture [Souza et al. 2019].

The contextual variability modeling creates software that is aware of contexts' changes to adapt its execution. The dynamics of contexts is inherently related to time and so, temporal information naturally guides the modeling of contextual changes. In this sense, three dimensions should be considered. The past has the collection of contexts experienced by an entity. This sequence of past contexts is called Contexts History [Rosa et al. 2015].

The present is the current relevant characteristics of an entity. The present is not only represented by the current context, but by the accumulated experience in the past contexts. The present of an entity is always changing, deriving from the past contexts. This dynamic description of an entity is its Profile which is captured through a strategy called Profile Management [Wagner et al. 2014]. The future is composed by contexts that have not yet occurred. Context Prediction [Rosa et al. 2016] uses different strategies to forecast likely contexts. This information is valuable to prepare a system to promptly adapt to complex situations that could not be processed in real time. 
A multi-temporal context-aware system [Rosa et al. 2015] uses the three dimensions to treat in an integrated way the contextual variability. The use of past, present and future allows the systems to make intelligent decisions based on complete information about users' contexts and their variability.

This article is organized in six sections. Section two discusses the aspects related to the past. Third section approaches the present, discussing aspect of profile management. Section four focuses on the future, mainly considering the context prediction. Section five proposes the system architecture to manage the multi-temporal context awareness. Finally, the last section presents conclusions and indicates directions of future works.

\section{The Past: Context History}

The storing and use of past information in computational systems is not new. On the other hand, the study of strategies to systematically store contexts for further use can be considered recent. One of the main applications of contexts history is to personalize services and contents according to the users' previous choices [Silva et al. 2010].

Figure 1 shows a context history in a context-aware competence management system [Rosa et al. 2015]. Contexts are stored in intervals forming a chronological sequence. The general idea is the same for all applications with only two differences. First, the interval at which the context is saved depends on the application. The contexts of a patient in an ICU should be stored more frequently than contexts of containers on a cargo ship. Second, the context model is specific of each application.

Figure 1 exemplifies a model to context-aware competence management system, even though, this model would be different for other domains. A current trend is to focus on well-defined domain representation to context models through Ontologies. The following challenges emerged:

- Is it feasible the use of a generic software framework to manage contexts histories? I believe the answer involves multi-domain ontologies of contexts and generic services to consult the histories [Silva et al. 2010];

- Can the storing of contexts be adaptive considering the contextual variability? Could the intervals and the contextual information be adapted according to the dynamic interests of the application? The recording of physiological signals should be accelerated if a critical situation was detected in a patient. Perhaps, the storing can be turned off at certain moments of the day, for instance, when the user goes to bed. How to model the strategy to adapt the storing of contexts? Can the contexts history already stored be used to decide the adaptation?

- What strategies do you use to manage a large database of contexts histories? This question assumes even more relevance when we consider that this database can store enormous amount of information, for example, complete lives of people or a large number of histories in smart cities. When and what contexts should be discarded? Maybe the best solution is not to discard contexts but compact them after some time. Nonetheless, if we access them, uncompressing the information will affect processing time. 


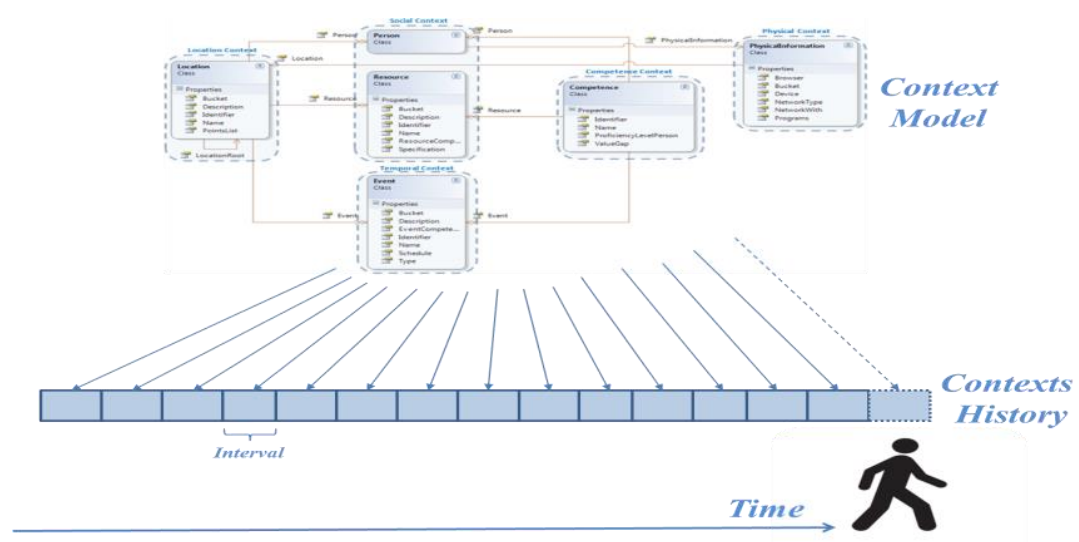

Figure 1. Contexts history management.

\section{The Present: Profile Management}

The present context of an entity can be instantly described by its current context. However, if we have its contexts history, the present representation can be richer because we can use the past to describe the present as a Profile [Wagner et al. 2014]. Profiles are dynamic summaries of relevant aspects inferred through the contexts history. Profiles are always updated and can be used to summarize contextual variability and to personalize the software. Figure 2 exemplifies the profile management through inferences rules updating aspects in a profile.

Personalization involves programs, which learn patterns, habits and preferences of a user, allowing the user modeling. This kind of application organizes its knowledge in User Profiles and the profiles are described in User Models. It is suitable to generate profiles through inferences on contexts histories, because histories are usually very dynamic, and inference rules propagate this dynamism to profiles. The current studies indicate some challenges:

- Is it feasible to use a generic framework to manage profiles? If so, systems could manage only the contexts histories and outsource the profile management [Wagner et al. 2014].

- How to explore the dynamicity provided by profiles based on contexts history? First, how often a manager should infer on contexts history to update the aspects of a profile? The aspects themselves can change based on rules used to update the user model. How to manage this aspects update?

- Can the contexts history be used to tune the refresh interval of a profile according the characteristics of the application?

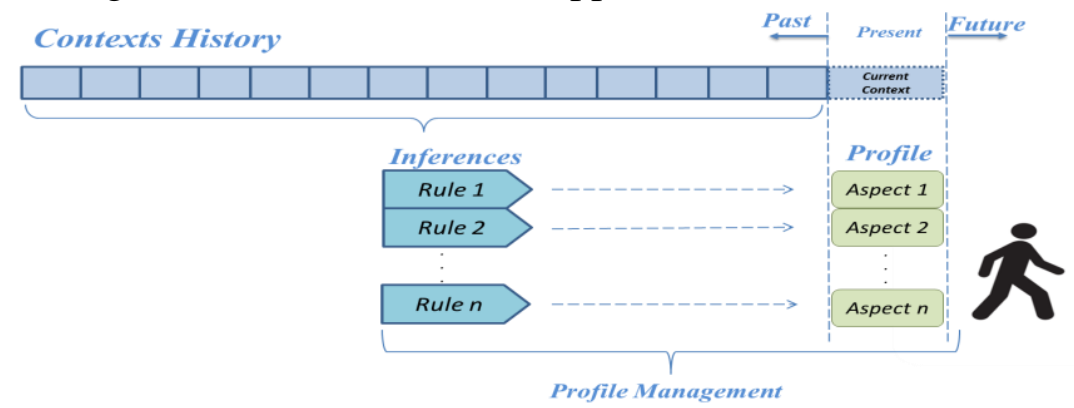

Figure 2. Profile Management using inference rules applied to contexts histories 


\section{The Future: Context Prediction}

If we can predict a future context of an entity, we can adapt the system to be prepared for specific situations. The use of contexts histories to infer future contexts is called Context Prediction [Rosa et al. 2016].

Prediction is not a new area in computing. The emergence of studies related to contexts histories has stimulated studies of context prediction. In the future, we will have large contexts histories of several entities and then, this information will allow a significant improvement in the context prediction. Besides, not only the history of an entity can be used to infer its future. If we have a great number of contexts histories, we can compare them to context prediction. In this sense, one of the most promising strategies is the similarity analysis of contexts histories [Wiedemann et al. 2016] (see Figure 3). Context prediction faces interesting challenges, such as:

- How to model the contexts histories to accelerate the prediction? Ontologies lighten the modeling and storage of contexts histories, though, the processing cost can make it unfeasible to compare a large number of histories during a real time similarity analysis;

- Is it possible to create a generic system to manage context prediction based on contexts histories, providing prediction services to other systems? If so, how can we treat the problem that there is no ideal strategy for all scenarios of prediction [Rosa et al. 2016]?

- The similarity analysis compares only two contexts histories. If we expand this strategy to compare several histories at same time, will it be possible to predict patterns and future trends of entity groups?

\section{Multi-temporal Context Awareness}

The integrated treatment of time dimensions allows a multi-temporal contextual variability modeling [Rosa et al. 2015]. A common sense is to consider the variability in the future direction, that is, contexts change as times goes by. However, if we have contexts histories, variability may be for the past, and we can make an entity return to an earlier context.

An intelligent airplane can detect that its current context is unsafe, returning to a previous context that it learned was safe in the current flight conditions. An audit system can return through a contexts history to determine in what context started the problem that is being audited. A multi-temporal context-aware system uses the time dimensions to adapt its execution to better achieve user goals.

Figure 4 shows a system architecture to manage the contextual variability on multitemporal systems. The applications interested in multi-temporal variability store locally their contexts history and regularly send this piece of history to the server. In addition, applications implement their engine using contextual variability services provided by the server. The server has modules to manage each time dimension (managers for the past [Silva et al. 2010], present [Wagner et al. 2014] and future [Rosa et al. 2016] [Wiedmann et al. 2016]) and a database to store profiles, complete contexts histories, context models and entity models. 


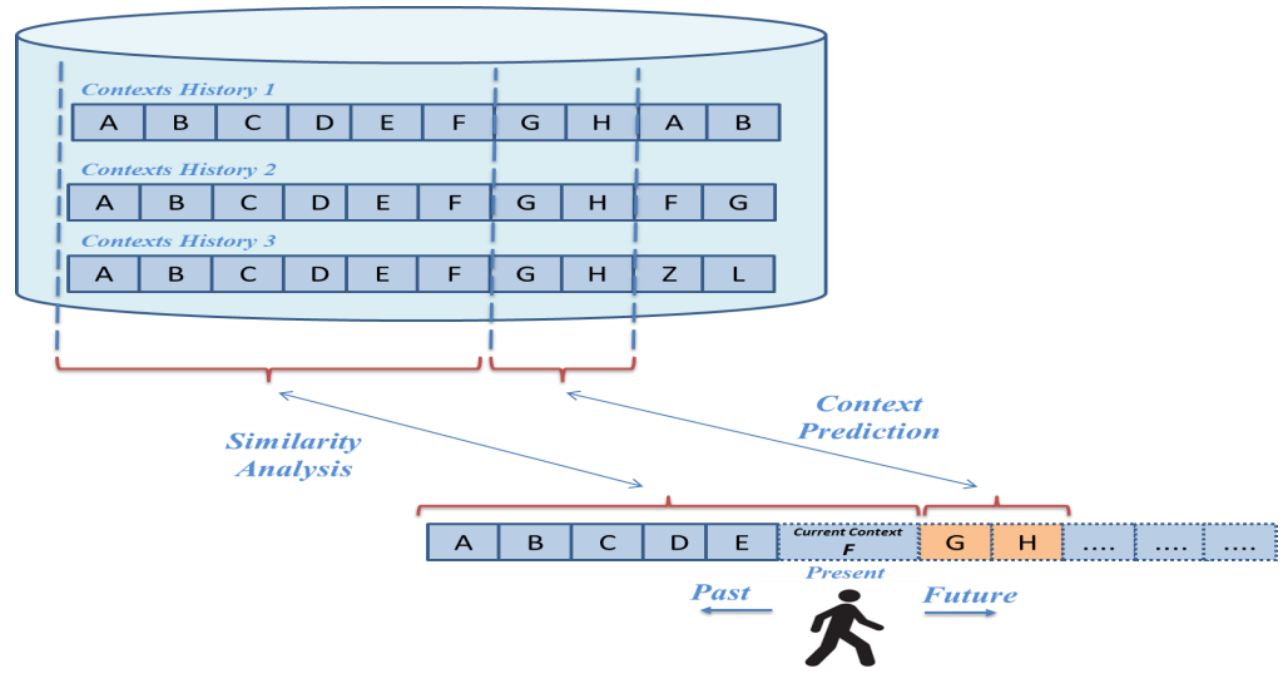

Figure 3. Profile Management using inference rules applied to contexts histories

The services implement generic functions to manage multi-temporal aspects, such as, register of entity models and context models, update of contexts histories and queries to obtain profiles, predictions, past contexts or pieces of contexts histories. Studies on multi-temporal systems are recent, so there are relevant challenges:

- How to model the return to the past using contexts histories in cases where contexts are related to the physical world which sometimes cannot be recovered?

- What performance problems emerge in a system like the one shown in figure 4 when we have several applications that require adaptation in real time?

- If inferences on contexts histories allow updating profiles, can context prediction be used to infer future profiles?

The integrated treatment of temporal dimensions in computer systems has always been considered a challenge. However, the consolidation of the context-aware systems allows the use of contexts as management unit to record the past, determine the present and predict the future. The modelling of multi-temporal contextual variability integrates the human perception of the time dimensions and the development of powerful adaptive systems. We are at the beginning of the challenge, but the history so far allows us to predict a promising future. The next subsections discuss the time dimensions and summarize the modules proposed to concrete each one of them.

\subsection{Contexts history manager (past)}

Rosa et al. [2016] discussed basic aspects of context awareness and strategies used to best use it. The authors argue that context-aware architectures which use not only present contexts, but also measurements of the past, need to store visited contexts for further use. This information from past contexts related to an entity is called its context history [Hong et al. 2009] [Ciaramella et al. 2010]. Some works refer to this history as trail [Silva et al. 2010] [Rigo et al. 2015].

Some works focus on life logging [Smith 2008] [Doherty et al. 2011] [Sellen and Whittaker 2010]. Their main purpose is to enhance human memory by using the capabilities of computers. Other works approach the use of contexts histories in the 
decision making process [Ciaramella et al. 2010] [Hong et al. 2009] [Baur et al. 2010]. Dey et al. [2001] also briefly described the importance of using history in decision.

The Context History Manager is based on the UbiTrail model [Silva et al. 2010]. Studies related to this model evaluated the value of considering users' past actions performed in the contexts visited during a period, such as, the activities done, the applications used, the contents accessed, and any other possible data [Rosa et al. 2015][Silva et al. 2010][Rigo et al. 2015]. This information helped to improve the distribution of content and services in context-aware environments, because applications were using an additional and more complete information source. In other words, applications started using contexts histories in conjunction with the current context information and users' profiles to take decisions [Wagner et al. 2014][Silva et al. 2010].

\subsection{Profile manager (present)}

Wagner et al. [2014] argued that for an application to adapt itself effectively to users, it is not only important to know the history of contexts visited by them, but to understand who the users are - that is, what are their relevant characteristics. This kind of application organizes its knowledge about a user in User Profiles [Waner et al. 2014]. The profiles are stored in a format called User Model [Fischer 2011].

Furthermore, the ubiquitous computing indicates that systems should become increasingly invisible to their users [Weiser 191]. However, a system can only become invisible if it is proactive, and can only be proactive by knowing the user [Satyanarayanan 2001]. Wagner et al. [2014] affirmed that for a system to know its users, it must know the user histories [Silva et al. 20108], understand the contexts in which they are inserted [Dey et al. 2001], and be able to process this set of information into user profiles [Wagner et al. 2014] [Fischer 2011].

In this sense, the Profile Manager is based on the eProfile model [Wagner et al. 2014]. This model is a ubiquitous profile manager of generic domain oriented towards distributed environments, with inter-system interoperability. eProfile infers profiles data through analysis of contexts histories of entities, following rules defined by the own entities, and provides data inferred to be used by different applications. These applications can register entities' actions in contexts histories and infer profile information from these histories, using semantic interoperability; thus allowing different applications to share information and infer a unified profile.

\subsection{Context prediction manager (future)}

Rosa et al. [2016] affirmed that with the use of the users' current contexts (present) and their contexts histories (past), applications already have a reasonable information source to base their decisions. Nonetheless, in order to become proactive and act before the context has actually changed, future contexts have to be predicted [Konig et al. 2011]. This has motivated researchers to study the use of another temporal aspect; the future [Sigg 2011]. The obtainment of the users' future contexts is made through predictions techniques. Based on users' histories and current contexts, algorithms predict the contexts that probably will describe the users' future situations [Sigg 2010].

Researches have explored prediction in various kinds of applications, such as trends of stocks [Ballings 2015] and failure in software [Bala and Chana 2015]. A research 
trend focuses on context prediction. Burbey and Martin [2012] organized and discussed works dedicated to predict personal mobility for location prediction. In addition, Lee and Lee [2012] use the location prediction to support ubiquitous decision support. Rosa et al. [2016] highlighted recent studies that discuss and classify works in research topics related to context prediction, such as anticipatory mobile computing [Pejovic and Musolesi 2015] and prediction in pervasive computing [Ameyed et al. 2015]. These surveys present and compare context prediction techniques and propose solutions, particularly highlighting challenges and opportunities associated with this research field. Pejovic and Musolesi [2015] indicate the treatment of privacy and anonymity as a challenge in research related to anticipatory mobile computing. Ameyed et al. [2015] state that another research challenge is the lack of general approaches for dealing with context prediction and, specifically, to allow automatic adaptation using context prediction.

The Context Prediction Manager is based on the Oracon model [Rosa et al. 2016]. Oracon is a generic model that adapts itself in order to apply the best prediction algorithm in a particular case and situation. This adaptive approach is the main contribution of this work and differentiates the proposed model of other related works. In addition, the model supports important features of ubiquitous computing, such as context formal representation and privacy, which are not well explored by other works. The main strengths of the proposed model are the flexibility allowed by the automatic selection of the prediction method and also the generic context information made possible by the formal representation. These features make the Oracon a generic model that can be used by any kind of context-aware application. On the other hand, its main weakness is related to performance constraints. Oracon selects the best prediction algorithm using a ranking obtained by testing all algorithms in a piece of contexts history provided by the application. This strategy may result in a high load processing and limit the feasibility of the proposal in real environments.

\section{Conclusion}

This article focused on a trend in context-aware systems, namely, the treatment of multitemporal aspects on contextual variability. The text discussed each time dimension, mainly indicating challenges related to each one. Besides, the article proposed an architecture to manage the contextual variability on multi-temporal systems.

The main contribution of this work is to highlight the challenges and trends in this emergent topic. Through this study, researchers can take the next steps in creating multitemporal systems based on contexts.

Future works can improve this study. Firstly, a systematic mapping study can be conducted to determine the researches related to context history focusing on research questions exploring as the works applied the time dimensions to solve problems using context-aware systems. A second work can apply the proposed architecture to solve real problems, allowing to evaluate the impact of this technology in computational environments. 


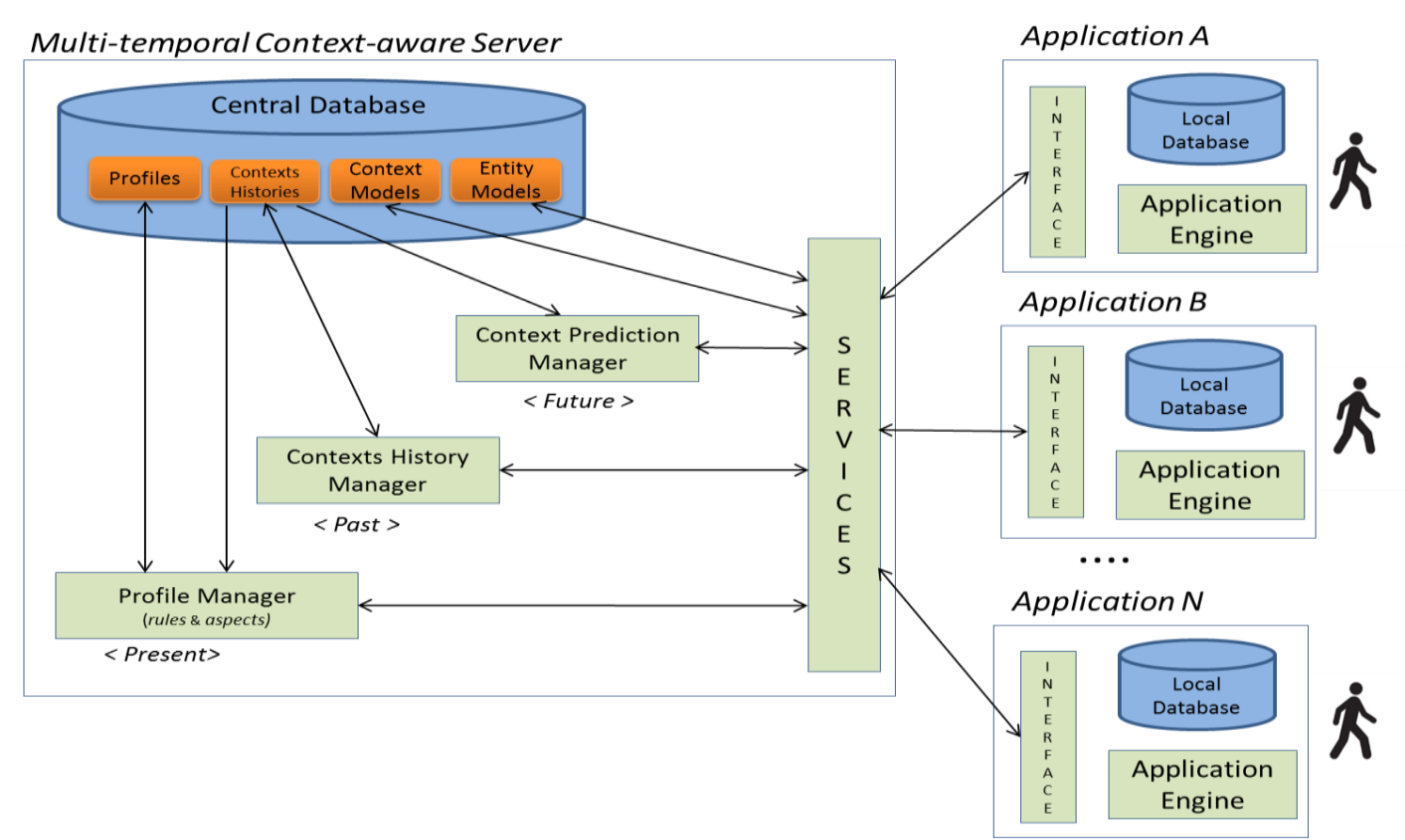

Figure 4: Model to manage the contextual variability on multi-temporal systems

\section{Acknowledgments}

The author wish to acknowledge that this work was supported by FAPERGS (Foundation for the Supporting of Research in the State of Rio Grande do Sul http://www.fapergs.rs.gov.br), CNPq (National Council for Scientific and Technological Development - http://www.cnpq.br) and Coordenação de Aperfeiçoamento de Pessoal de Nível Superior (CAPES) - Finance Code 001. I especially thank the Unisinos (University of Vale do Rio dos Sinos - http://www.unisinos.br) and the Mobilab (Mobile Computing Lab - http://www.unisinos.br/mobilab) for embracing this research. Finally, I thank the organizing committee of the VIII SBCUP (2016) for the invitation to give the opening speech titled "UbiComp \& Interdisciplinaridade: Ubiquidade, ZenBudismo e Computação" (https://goo.gl/6bkH4y). The meeting was a moment of inspiration to discuss space, time, context awareness and Ubiquitous Computing.

\section{References}

Ameyed, D., Miraoui, M. and Tadj, C. (2015) "A Survey of Prediction Approach in Pervasive Computing", International Journal of Scientific \& Engineering Research, 6(5), p.1-11.

Bala, A. and Chana, I. (2015) "Intelligent failure prediction models for scientific workflows". Expert Systems with Applications. 42(3), p.980-989. http://dx.doi.org/10.1016/j.eswa.2014.09.014

Ballings, M., Van den Poel, D., Hespeels, N. and Gryp, R. (2015). "Evaluating multiple classifiers for stock price direction prediction". Expert Systems with Applications. 42(20), p.7046-7056. http://dx.doi.org/10.1016/j.eswa.2015.05.013

Barbosa, J., Martins, C., Franco, L. and Barbosa, D. (2016) "TrailTrade: a model for trail-aware commerce support", Computers in Industry, 80, p.43-53. http://dx.doi.org/10.1016/j.compind.2016.04.006 
Barbosa, J., Tavares, J., Cardoso, I., Mota, B. and Martini, B. (2018). "TrailCare: na Indoor and Outdoor Context-aware System to Assit Wheelchair Users". Internacional Journal of Human-Computer Studies. 116, p.1-14. https://doi.org/10.1016/j.ijhcs.2018.04.001

Burbey, I. and Martin, T. L. (2012) "A Survey on Predicting Personal Mobility". International Journal of Pervasive Computing and Communications. 8(1), p.5-22. http://dx.doi.org/10.1108/17427371211221063

Baur, D., Seiffert, F., Sedlmair, M. and Boring, S. (2010) "The streams of our lives: Visualizing listening histories in context". IEEE Transactions on Visualization and Computer Graphics. 16(6), p.1119-1128. http://dx.doi.org/10.1109/TVCG.2010.206

Ciaramella, A., Cimino, M. G. C. A., Lazzerini, B. and Marcelloni, F. (2010) "Using context history to personalize a resource recommender via a genetic algorithm", in Proceedings of the 10th International Conference on Intelligent Systems Design and Applications. Cairo, Egypt, p.965-970. http://dx.doi.org/10.1109/ISDA.2010.5687064

Dey, A., Salber, D. and Abowd, G. (2001). "A conceptual framework and a toolkit for supporting the rapid prototyping of context-aware application", Human-Computer Interaction, 16(2), p. 97-166. http://dx.doi.org/10.1207/S15327051HCI16234_02

Doherty, A. R., Caprani, N., Conaire, C. O., Kalnikaite, V., Gurrin, V. C., Smeaton, A. F. and O'Connor, N. E. (2011) "Passively recognising human activities through lifelogging". Computers in Human Behavior. 27(5), p.1948-1958. http://dx.doi.org/10.1016/j.chb.2011.05.002

Fischer, G. (2011) "User modeling in human-computer interaction. User Modeling and User-Adapted Interaction”. 11(1), p.65-86. http://dx.doi.org/10.1023/A:1011145532042

Hong, J., Suh, E.-H., Kim, J. and Kim, S. (2009) "Context-aware system for proactive personalized service based on context history". Expert Systems with Applications. 36(4), p.7448-7457. http://dx.doi.org/10.1016/j.eswa.2008.09.002

Konig, I., Voigtmann, C., Klein, B. N. and David, K. (2011) "Enhancing alignment based context prediction by using multiple context sources: experiment and analysis", in Proceedings of the 7th International and Interdisciplinary Conference on Modeling and Using Context, Karlsruhe, Germany, p.159-172. http://dx.doi.org/10.1007/978-3-642-24279-3_18

Larentis, A., Barbosa, J., Barbosa, D., Silva, C. and Barbosa, J. (2019) “Applied Computing to Education on Noncommunicable Chronic Diseases: A Systematic Mapping Study", Telemedicine and e-Health, 1, p.1-10, 2019. https://doi.org/10.1016/j.ipl.2019.03.010

Lee, S. and Lee, K. C. (2012) "Context-prediction performance by a dynamic Bayesian network: Emphasis on location prediction in ubiquitous decision support environment". Expert Systems with Applications. 39(5), p.4908-4914. http://dx.doi.org/10.1016/j.eswa.2011.10.026

Pejovic, V. and Musolesi, M. (2015) "Anticipatory Mobile Computing: A Survey of the State of the Art and Research Challenges". ACM Computing Surveys. 47(3), article n. 47. http://dx.doi.org/10.1145/2693843

Rigo, S., Cambruzzi, W. and Barbosa, J. (2015) "Dropout Prediction and Reduction in Distance Education Courses with the Learning Analytics Multitrail Approach", Journal of Universal Computer Science, 21(1), p.23-47. http://dx.doi.org/10.3217/jucs-021-01-0023 
Rosa, J., Barbosa, J., Kich, M. and Brito, L. (2015) "A Multi-Temporal Context-aware System for Competences Management", International Journal of Artificial Intelligence in Education,25(4), p.455-492.http://dx.doi.org/10.1007/s40593-015-0047-y

Rosa, J., Barbosa, J. and Barcelos, G. (2016) "ORACON: An Adaptive Model For Context Prediction", Expert Systems with Applications, 45(1), p.56-70. http://dx.doi.org/10.1016/j.eswa.2015.09.016

Satyanarayanan, M. (2001) "Pervasive computing: vision and challenges", IEEE Personal Communications, 8(4), p. 10-17. http://dx.doi.org/10.1109/98.943998

Sellen, A. J. and Whittaker, S. (2010) "Beyond total capture: a constructive critique of lifelogging", Communications of the ACM. 53(5), p.70-77. http://dx.doi.org/10.1145/1735223.1735243

Silva, J., Rosa, J., Barbosa, J. Barbosa, D. and Palazzo, L. (2010) "Content Distribution in Trail-aware Environments", Journal of the Brazilian Computer Society", 16(3), p.163-176. http://link.springer.com/article/10.1007/s13173-010-0015-1

Sigg, S., Haseloff, S., and David, K. (2010). "An alignment approach for context prediction tasks in ubicomp environments". IEEE Pervasive Computing. 9(4), p.9097. http://dx.doi.org/10.1109/MPRV.2010.23

Sigg, S. et al. (2011) "Investigation of context prediction accuracy for different context abstraction levels". IEEE Transactions on Mobile Computing. 11(6), p.1047-1059. http://dx.doi.org/10.1109/TMC.2011.170

Smith, S. (2008) "Who controls the past controls the future - life annotation in principle and practice", University of Southampton, School of Electronics and Computer Science, $\mathrm{PhD}$ Thesis. http://expertise.ecs.soton.ac.uk/16554/1/thesis.pdf

Souza, R., Lopes, J., Geyer, C., João, L., Cardozo, A., Yamin, A., Gadotti, G. and Barbosa, J. (2019). "Continuous Monitoring Seed Testing Equipaments Using Internet of Things", Computers and Electronics in Agriculture, 158, p.122-132. http://dx.doi.org/10.1016/j.compag.2019.01.024

Vianna, H. and Barbosa, J. (2014) "A Model for Ubiquitous Care of Noncommunicable Diseases", IEEE Journal of Biomedical and Health Informatics, 18(5), p. 1597-1606. http://dx.doi.org/10.1109/JBHI.2013.2292860

Vianna, H. and Barbosa, J. (2017) "In the Pursuit of Hygge Software", IEEE Software, 34, p. 48-52. https://doi.org/10.1109/MS.2017.4121208

Vianna, H. and Barbosa, J. (2019) "A scalable model for building context-aware applications for noncommunicable diseases prevention", Information Processing Letters, p.1-10. http://dx.doi.org/10.1016/j.ipl.2019.03.010

Wagner, A., Barbosa, J. and Barbosa, D. (2014) "A Model for Profile Management Applied to Ubiquitous Learning Environments", Expert Systems with Applications, 41(4), p. 2023-2034. http://dx.doi.org/10.1016/j.eswa.2013.08.098

Weiser, M. (1991) “The Computer for the 21st Century”, Scientific American, 265(3), p. 94-104. http://dx.doi.org/10.1145/329124.329126.

Wiedemann, T., Barbosa, J., Rigo, S. and Barbosa, D. (2016) "RecSim: A Model for Learning Objects Recommendation using Similarity of Sessions", Journal of Universal Computer Science, 22(8), p.1175-1200. http://www.jucs.org/jucs_22_8/recsim_a_model_for 